\title{
Procedure for TEM Measurement of Nanoparticles
}

\author{
William G. Stratton, Stuart McKernan, and Mary I. Buckett \\ 3M Corporate Research Analytical Laboratory, 3M Center, St. Paul, MN, 55144 USA
}

Nanotechnology has impacted a number of core technology platforms at $3 \mathrm{M}$, as well as other companies, and has been leveraged to produce game-changing new product opportunities. A subset of nanotechnology, nanoparticles, is used in these products and thus requires solid knowledge of both the nanoparticle size and distribution. As particles get smaller, classic particle sizing techniques like dynamic light scattering (DLS) do not have the ability to give an accurate size distribution of nanoparticles, and therefore TEM is being more commonly utilized for accurate nanoparticle sizing [1].

When initially sizing nanoscale items, it is always prudent to use multiple techniques. For example, combining DLS and TEM ensures that larger particles and smaller particles are both identified. Once sufficient material has been analyzed, you can usually proceed with a single technique and occasional spot checks with additional techniques to ensure the quality of the starting material has not changed.

The first step in preparing TEM samples is achieving the dilution ratio of nanoparticles to diluant to achieve a monolayer of nanoparticles visible on the sample grid when viewing in the microscope. More than a monolayer of nanoparticles creates too much nanoparticle overlap to accurately view particle edges for measurement. Once the dilution ratio is set, sonication of the solution is helpful to break up nanoparticle agglomerates (if appropriate for the sample type).

It is a natural tendency of microscopists to identify areas of highest particle density, to collect the most information possible from each image. However, to avoid bias sampling, all imaging areas should be taken at random regardless of the number of nanoparticles in the field of view. A natural agglomeration of particles can occur as the water dries off the sample causing particle movement based on size. It is important to image several areas of the grid to ensure imaging of as many different sizes and shapes of nanoparticles in the sample as possible. It is also important to keep the average number of counts approximately the same between images, especially when using an automated method for particle measurement.

Particle size and distribution measurements can utilize an automated particle identification routine (e.g. ImageJ) or manual measurements. Most automated routines use thresholding to identify the different particles; therefore automated routines tend to work better when there is good contrast between the particles and the background. This tends to be the situation when viewing larger particles (greater than $50 \mathrm{~nm}$ ). Also, several automated sizing routines work best with simple particle shapes like spheres versus more complex shapes or samples which are heavily agglomerated. Furthermore, there can be misidentification of particles with automated routines. It is always a good idea to get an overlay of identified particles to the actual TEM image to ensure appropriate correspondence between the computer and the user to remove any ambiguity of what is being measured, as seen in Figure 1. 
Manual measurements tend to take more time, but can be applied to more situations than automated methods. Generally manual methods are most useful for small particles that have low contrast between the particle and the image background where thresholding and automatic routines cannot consistently identify the particles. There are other circumstances where manual measurements work best, like heavily agglomerated particles (where automated routines cannot identify individual particles) or particles with odd shapes. Also, as in automated routines, it is best to include images with overlaid manual measurements to remove ambiguity of what was measured (Figure 1)

Regardless of measurement type, it is critical to know how many measurements are needed to get a representative sampling of the nanoparticles. There are several resources that provide information on counting statistics; in our analytical laboratory we follow ASTM E122, "Standard Practice for Calculating Sample Size to Estimate,With Specified Precision, the Average for a Characteristic of a Lot or Process" [2].

ASTM E122 assumes a normal distribution of the nanoparticles and relates the measured standard deviation and to an acceptable error to determine the needed number of measurements. Since the standard deviation can change with additional measurements, the number of required measurements can also change. To increase the efficiency in an analytical laboratory setting, we place the formula in a spreadsheet with our nanoparticle measurements to quickly relate the number of measurements taken to the needed number of measurements to satisfy the acceptable error. Results of standard statistical measures (e.g. mean, standard deviation, taken measurements etc.) and a histogram are reported and archived.

In conclusion, we review the sample preparation methods for preparing, imaging, and measuring nanoparticle samples to give a reasonable estimation of the nanoparticle size and distribution. By using ASTM E122 we have a standardized and repeatable method for determining the needed number of measurements to provide a size distribution with a provided acceptable error.
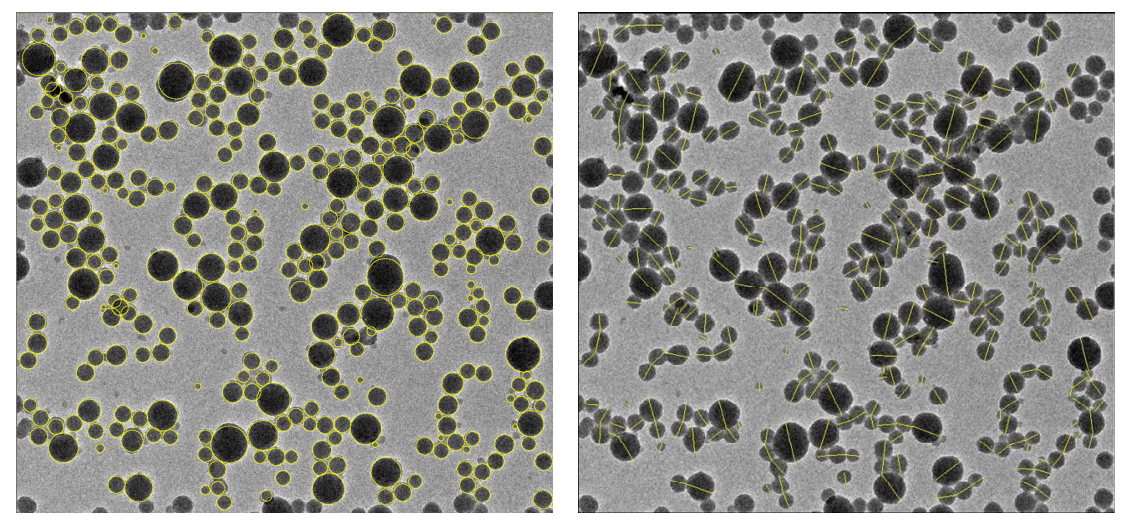

Figure 1: Automated (left) and manual (right) measurements overlaid on a TEM image

References:

[1] G. Orts-Gil, K. Natte, D. Drescher, J. Nanopart. Res 13 (2011), p. 1593

[2] ASTM Standard E122-09E1, 2011, "Standard Practice for Calculating Sample Size to

Estimate,With Specified Precision, the Average for a Characteristic of a Lot or Process" ASTM

International, West Conshohoken, PA, 2003, DOI: 10.1520/C0033-03, www.astm.org 